\title{
Embedded filter bank-based algorithm for ECG compression
}

\author{
Manuel Blanco-Velasco , Fernando Cruz-Roldán , Eduardo Moreno-Martínez , \\ Juan-Ignacio Godino-Llorente, Kenneth E. Barner \\ Department of Teoria de la Señal y Comunicaciones, Universidad de Alcala, Campus Universitario, 2887I Alcala de Henares, Madrid, Spain \\ Department of Ingenieria de Circuitos y Sistemas, Unicersidad Politécnica de Madrid, Madrid. Spain \\ Department of Electrical and Computer Enginering, Unicersity of Deloware, Newark, DE 197I6, USA
}

\begin{abstract}
In this work, two ECG compression schemes are presented using two types of filter banks to decompose the incoming signal: wavelet packets (WP) and nearly-perfect reconstruction cosine modulated filter banks. The conventional embedded zerotree wavelet (EZW) algorithm takes advantage of the hierarchical relationship among subband coefficients of the pyramidal wavelet decomposition. Nevertheless, it performs worse when used with WP as the hicrarehy becomes more complex. In order to address this problem, we propose a new technique that considers no relationship among coefficients, and is therefore suitable for use with WP. Furthermore, this new approximation makes it possible to apply the quantization method to $M$-channel maximally decimated filter banks. In this fashion, the proposed algorithm provides two efficient and effective ECG compressors that show better ECG compression performance than the conventional EZW algorithm.
\end{abstract}

Keywords: Electrocardiogram (ECG); ECG compression; Embedded zerotree wavelet; Wavelet packets (WP); Channel bank filter; Filtering theory; Filter bank

\section{Introduction}

The electrocardiogram (ECG) depicts the electrical activity of the heart. ECG processing is a topic of great interest within the scientific community because of two reasons: (1) ECG is collected by non invasive means, which allows easy and wide availability and (2) the ECG contains very much information, which is highly valuable for diagnosing. In 1961, Holter introduced the long term recording ECG in ambulatory patients as a new tool for certain pathologies. These type of records are commonly used and they are typically collected over $24 \mathrm{~h}$, which increases the volume of data. Besides, ECG is also essential to monitoring patients at home advancing telemedical applications . For these cases, compression is needed to provide either transmission or storage solutions for reducing the original bit rate. Therefore, the design of ECG compression techniques has been widely studied in the last few years. A classification in three categories of the most common techniques was proposed : direct methods, transform methods and other compression methods. 
The use of wavelets in ECG analysis was first introduced. From this initial effort, a wide variety of ECG wavelet-based compressors have been proposed where compression schemes are carried out utilizing the discrete wavelet transform (DWT) . The embedded zerotree wavelet (EZW) algorithm was specifically designed to use the DWT [13] in image coding applications. This method demonstrated good performance and was quickly applied to other types of signals, such as ECG and myoelectric signals. The embedded property of the EZW algorithm allows the bits in the bit stream to be arranged in 'order of importance' so that the encoding process can be stopped at any point once the target is achieved.

In the DWT decomposition algorithm, every coefficient at any scale is related with two other coefficients at the immediate lower scale. This correspondence is iterated through scale, giving the temporal orientation tree. An example is illustrated in Fig. 1. The set of a coefficient and its descendents are called zerotree. In the encoding process, the whole set of coefficients of a zerotree can be referenced by its root, which is the first coefficient of the temporal orientation tree at the lower scale. Also, a coefficient is called significant if its magnitude is greater than a given threshold value $\varepsilon$. Therefore, depending on the magnitude of a coefficient related to $\varepsilon$, i.e., its significance, it can be encoded as a symbol of a reduced alphabet to obtain a significance map. The EZW algorithm takes into account the hierarchy of the DWT coefficients among different subbands to efficiently encodes the significance map and use an alphabet of four symbols: $\{$ POS, NEG, IZ, ZTR $\}$. Symbols $\{P O S\}$ and [NEG\} indicate the sign of a significant coefficient. A non-significant coefficient is encoded with the symbol [ZTR\} if it is the root of a zerotree where all its coefficients are non-significant. Con- versely, a non-significant coefficient is encode as an isolate zero with the symbol $\{I z\}$.

In the ECG compression cases, the EZW algorithm is reported using wavelet packets (WP). The performance of this approach is worse than the DWT-based algorithm. The reason for the poor performance in the WP case is that the best basis decomposition often splits the signal into a number of smaller hierarchies that cannot be efficiently encoded by zerotrees.

The first motivation of this work has been the development of an EZW-based algorithm to be used with WP, improving the performance of the previously reported methods. So, the hierarchical relationships among coefficients is not taken into consideration. The consequence of this is that the \{ZTR\} symbol that identifies the root of a zerotree can be withdrawn from the alphabet so that only three symbols ( $\{$ POS, NEG, IZ\}) are needed to encode the significance map.

The second goal of this work is to use a modulated $M$-channel maximally decimated filter bank in the encoder, since it reported good performance in ECG compression. For example, a low computational complexity algorithm applied with nearly-perfect reconstruction cosine modulated filter banks (N-PR CMFB) is proposed in [11]. An important contribution of this work is that the proposed algorithm can also be used with these types of filter banks as a result of its versatility based on the non-use of hierarchical relationships among subbands. To our knowledge, the N-PR CMFB were used for the first time, where good results are reported in the comparison against the DWT.

Therefore, in this paper we present two versatile embedded encoding schemes: (1) the embedded wavelet packets (EWP) algorithm to be used with WP and (2) the embedded filter banks (EFBs) algorithm to be used with N-PR CMFB.

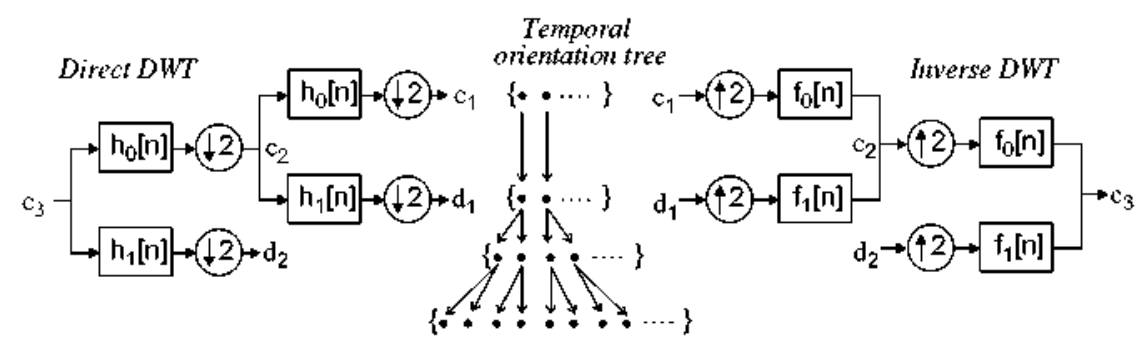

Fig. 1. Algorithm for the computation of DWT. Both $h_{0}[n]$ and $f_{0}[n]$ are low-pass filters, while $h_{1}[\eta t]$ and $f_{1}[n]$ are high-pass filters. A temporal orientation tree scheme is depicted showing the relationships anong coefficients through scale. 
Simulations results are provided demonstrating the improvement of the proposed encoders over the original EZW DWT-based algorithm. Finally, it should be emphasized that although this work focuses on ECG, other signals, such as myoelectric signals or images (processed using linear-phase filter banks), can also be compressed with the proposed algorithms.

The remainder of this paper is structured as follows. In Section 2 the decomposition methods used in this work are presented. In Section 3, the compression algorithms are detailed. The results and discussion are shown in Section 4 and finally, the conclusions are given in Section 5.

\section{Review of decomposition methods}

\subsection{Watelets packets}

The DWT decomposes a signal $f(t)$ as a successive approximation in several scales as follows [15]:

$f(t)=\sum_{k} c_{j_{0}}(k) \varphi_{j_{0}, k}(t)+\sum_{k} \sum_{j=j_{0}}^{\infty} d_{j}(k) \psi_{j, k}(t)$,

where bases functions are dilated and translated versions of the wavelet $\psi(t)$ and the scaling $\varphi(t)$ functions:

$\psi_{i, k}(t)=2^{j / 2} \psi\left(2^{j} t-k\right)$,

$\varphi_{j, k}(t)=2^{j / 2} \varphi\left(2^{j} t-k\right)$.

The coarse details of $f(t)$ are represented by the scaling coefficients $c_{j_{0}}(k)$ while the finer details are represented by the wavelets coefficients $d_{j}(k)$. The DWT is efficiently computed by 2-channel perfect reconstruction (PR) filter banks iteratively applied to the low-pass channel as shown in Fig. 1, where the number of layers or levels of the resulting filter bank depends on the desired resolution scale. The set of DWT coefficients are given at the outputs of the direct transform side when the incoming samples are the scaling coefficients of $f(t)$ at a higher scale. The inverse transform is carried out with the corresponding synthesis filter bank.

WP theory is a generalization of DWT. The input signal is decomposed applying the 2-channel PR filter bank at both the low and high-pass branch. The resulting binary tree is considered as a library of bases of which only one would be needed to represent the incoming signal. The number of bases

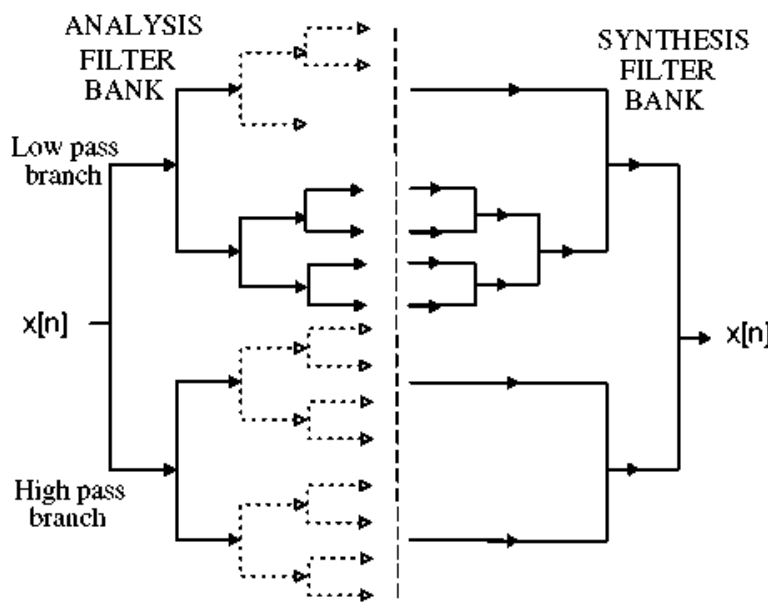

Fig. 2. Exanple of WP for a depth of four layers. The broken lines correspond with the pruned branches.

$A_{n}$ for an $n$-layers WP can be recursively calculated as

$A_{n}=1+A_{n-1}^{2}$,

where $A_{n-1}$ is the number of bases of an $(n-1)$ layers WP. Therefore, WP can be adaptively utilized by selecting the best base, which consists of pruning the tree according to a cost function. The best base selection algorithm used in this work is that proposed An example of best base selection for a 4-layer WP is shown in Fig. 2. Basically, the whole binary tree is first obtained and subsequently pruned according to the Shannon entropy as proposed. The broken lines in Fig. 2 correspond to the rejected branches, while the others give the filter bank for processing the incoming signal. Accordingly, different filter banks are used when the input signal is split into blocks.

\subsection{Nearly-perfect reconstruction cosine modtlated fliter banks}

An N-PR CMFB is a subclass of modulated $M$-channel maximally decimated filter bank whose structure is shown in Fig. 3. All the analysis $h_{k}[n]$ and synthesis $f_{k}[n]$ filters, $0 \leqslant n \leqslant(L-1)$, $0 \leqslant k \leqslant(M-1)$, can be obtained through the modulation of a low-pass prototype filter $p[n]$ as follows:

$h_{k}[n]=2 \cdot p[n] \cdot \cos \left((2 k+1) \frac{\pi}{2 M}\left(n-\frac{L}{2}\right)+(-1)^{k} \frac{\pi}{4}\right)$, 


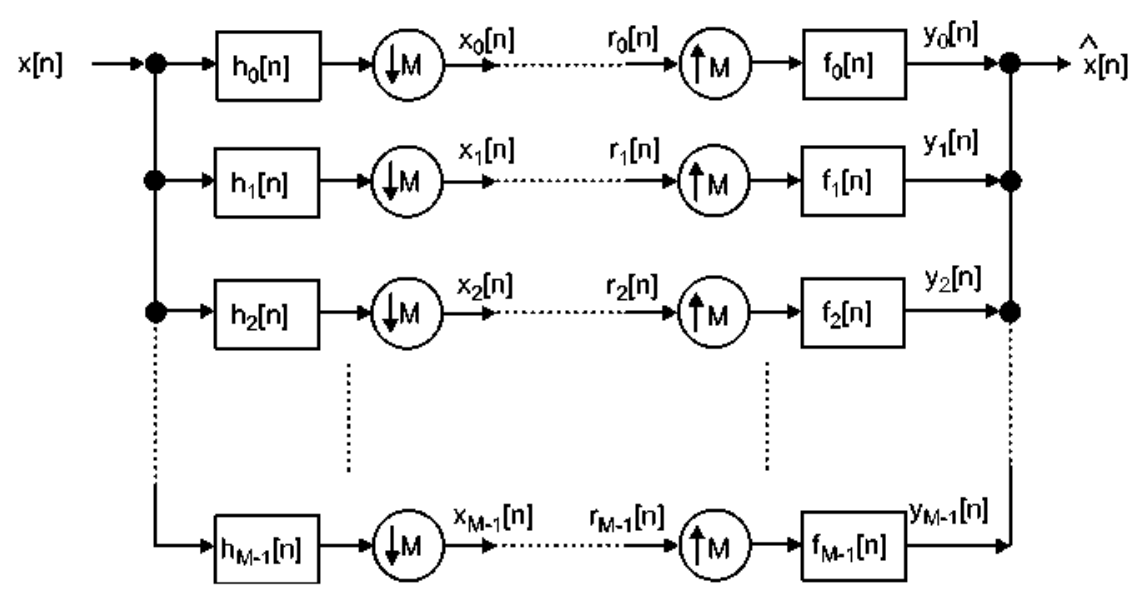

Fig. 3. $M$-channel maximally decimated filter bank.

$f_{k}[n]=2 \cdot p[n] \cdot \cos \left((2 k+1) \frac{\pi}{2 M}\left(n-\frac{L}{2}\right)-(-1)^{k} \frac{\pi}{4}\right)$.

In this work, the prototype filter is designed with the technique proposed This technique offers almost, but not true, PR, and controls the position of the $3 \mathrm{~dB}$ cutoff frequency of the prototype filter, setting it approximately at frequency $\omega=\pi / 2 M$. The problem can be stated several ways, but the goal is minimizing

$\phi=|| P\left(\mathrm{e}^{\mathrm{j} \pi /(2 M)}\right)|-1 / \sqrt{2}|$,

where $P\left(\mathrm{e}^{\mathrm{j} \pi /(2 M)}\right)$ is the frequency response of the prototype filter $P\left(\mathrm{e}^{\mathrm{j} \omega}\right)$ for $\omega=\pi /(2 M)$. When using an appropriate FIR filter design technique (by windowing or by means of the Parks-McClellan algorithm), it is guaranteed that the frequency response of the prototype filter approximately satisfies the power complementary property. In this way, it is possible to reduce the amplitude distortion and the aliasing error introduced in the filter bank.

The interest of using CMFB is based on the fact that it can be efficiently implemented by means of polyphase structures that considerably reduce the computational complexity . , we present a detailed study of the computational complexity for N-PR CMFB applied to ECG compression. Furthermore, the use of N-PR instead of PR systems is inspired by the fact that, for a previously fixed filter length, higher selectivity and discrimination systems can be obtained designing the former one (N-PR).

\section{Proposed algorithms}

In this section, we present two compression schemes that differ in the decomposition techniques used to represent the incoming signal. Here, the details of the encoding scheme are explained given that the incoming signal has been decomposed. These compressors do not need any signal preprocessing such as QRS complex detection and no a priori signal knowledge is required. Both methods work over non-overlapped blocks of $N$ samples of the incoming signal.

\subsection{Embedded wavelet packets (EWP) algorithm}

The WP-based method consists of the following steps:

(1) Every input block of $N$ samples is decomposed using WP. For this purpose, the best base of every block is obtained by means of the pruning algorithm proposed in the prior section.

(2) The coefficients are encoded with an EZWbased embedded algorithm.

(3) The significance map is entropy encoded.

The embedded algorithm is carried out as successive approximations that are applied to each group of $N$ coefficients obtained from the corresponding $N$ incoming samples. Successive thresholds $\varepsilon_{i}=2^{p-i}$, $p \in \mathbb{Z}, i=0,1, \ldots, L$, are iteratively applied to obtain significance maps associated to each threshold. Let $\left\{c_{i}\right\}, \forall i=1, \ldots, N$ be the set of WP 
coefficients; the first threshold value is set as

$p=\left\lfloor\log _{2}\left(\max _{c \in\left\{c_{i}\right\}}\left\{\left|c_{i}\right|\right\}\right)\right\rfloor$.

where $\lfloor\cdot\rfloor$ denotes rounding to the next smaller integer. Two lists must be maintained while the encoding (decoding) process proceeds: The dominant list (DL) contains all the coefficients found not significant to the current and prior thresholds, and the subordinate list (SL) contains the magnitudes of the coefficients found significant. Initially, DL equals the coefficients resulting of the transform of the corresponding incoming block, and $\mathrm{SL}$ is empty. These lists are updated at every iteration.

The dominant pass and the subordinate pass are accomplished for every threshold. During the dominant pass, coefficients in DL are compared with the threshold, e.g., $\varepsilon_{0}=2^{p}$ for the first iteration. Then, the significance map is encoded as is explained in Fig. 4, with a three symbols alphabet: \{POS, NEG, IZ\}. The magnitude of the significant coefficients (encoded either as $\{P O S\}$ or $\{\mathrm{NEG}\}$ ) is included in SL. Subsequently, the significant coefficients are zeroed in DL to avoid being significant at the following iteration. The reconstructed magnitude of a significant coefficient, for the threshold $2^{p}$ case, on the decoder side is $\left|\hat{c}_{i}\right|=2^{p}+2^{p-1}$. The sign

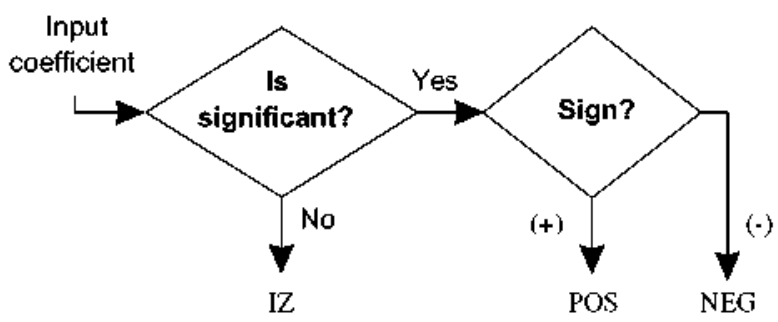

Fig. 4. Alphabet for the significance map. is taken from the corresponding code in the significance map.

The subordinate pass runs after the dominant pass has finished. The aim is to make all the previous significant coefficients (those included in SL) one bit more accurate. For instance, Fig. 5 shows the subordinate pass applied three consecutive times to a coefficient, which is significant to the threshold of the second iteration (for $p-1$ ). Its initial value is $\left|\hat{c}_{i, 1}\right|=2^{p-1}+2^{p-2}$. There is an uncertainty interval associated with the current threshold whose width is $2^{p-2}$. The actual coefficient value is within the upper half of the uncertainty interval, so a ' 1 ' is assigned as refinement bit and the reconstructed coefficient value is $\left|\hat{c}_{i, 2}\right|=\left|\hat{c}_{i, 1}\right|+2^{p-2}$. In Fig. 5, the updated value, the first time that the subordinate pass is applied, is designated by the curved solid line arrow. In the following iteration (for $p-2$ ), the uncertainty interval width is $2^{p-3}$ and $a$ ' 0 ' is the assigned refinement bit that indicates that the actual coefficient value is in the lower half, yielding the reconstructed coefficient $\left|\hat{c}_{i, 3}\right|=$ $\left|\hat{c}_{i, 2}\right|+2^{p-3}$. Once again, for the following iteration, the refinement bit is ' 0 ' so the coefficient takes value $\left|\hat{c}_{i, 4}\right|=\left|\hat{c}_{i, 3}\right|+2^{p-4}$. All the coefficients found in the SL are refined as above in each iteration. To do so, the encoder generates the refinement list (RL), which contains the refinement bits to be used by the decoder.

The encoding and decoding processes are summarized as follows. From the encoder side, let $\left\{c_{i}\right\}, \forall i=1, \ldots, N$ be the set of WP coefficients, the encoding algorithm works as follows:

Step 1. Output $p=\left\lfloor\log _{2}\left(\max _{i \in\left\{c_{i}\right\}}\left\{\left|c_{i}\right|\right\}\right)\right\rfloor$.

Step 2. Intialization of lists:

(a) $D L=\left\{c_{i}\right\}, \forall i=1, \ldots, N$.

(b) $S L=\{\phi\}$.

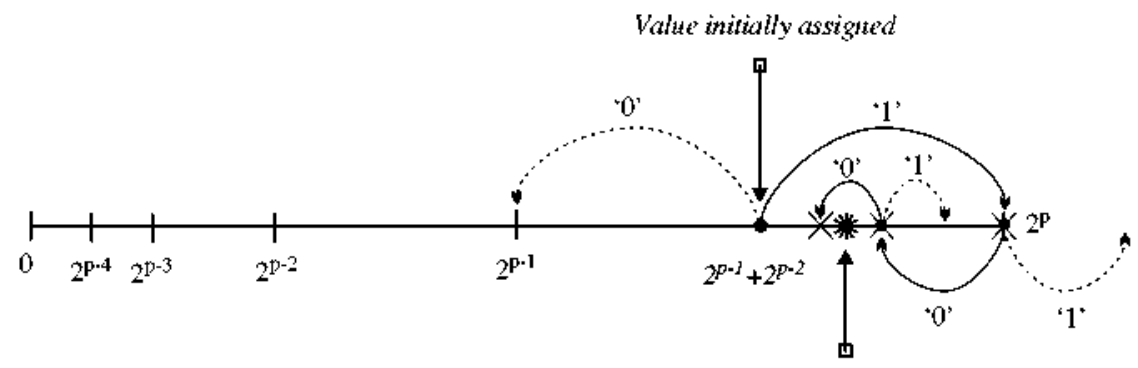

Coefficient value

Fig. 5. Example of successive refinement of a coefficient. The curved solid line arrows point at the final values once the refinement bit is successively assigned. 
Step 3. Dominant pass:

(a) $D L(i), \forall i=1, \ldots, N$, is encoded as in Fig. 4.

(b) If $D L(i)$ is significant, its magnitude is inchuded in $S L$ and $D L(i)=0$.

Step 4. Subordinate pass: the refinement list is generated.

Step 5. $p=p-1$ and go to Step 3 .

Conversely, the decoder performs as follows:

Step 1. Initialization of lists:

(a) $D L=\left\{c_{i}\right\}, c_{i}=0, \forall i=1, \ldots, N$.

(b) $S L=\{\phi\}$.

Step 2. Initial threshold $p$ is received.

Step 3. Dominant pass:

(a) $\{$ POS $\}: D L(i)=2^{p}+2^{p-1}$ and its magnitude is inchided in SL.

(b) $\{\mathrm{NEG}\}: D L(i)=-\left(2^{p}+2^{p-1}\right)$ and its magnitude is included in $\mathrm{SL}$.

(c) $\{\mathrm{IZ}\}$ : nothing is done.

Step 4. Subordinate pass: coefficients in SL are refined after receiving $\mathrm{RL}$.

Step 5. $p=p-1$ and go to Step 3 .

The encoding-decoding process stops when some target is reached, such as the quality of the retrieved signal, the compression ratio (CR), or a specific number of iterations.

Giving the above, the stream for every incoming block consists of a header of $B$ bits followed by groups of bit sets equal to the number of approximations made for the corresponding segment, as is shown in Fig. 6, where a number of $L$ iterations have been achieved. For every iteration, the significance map and the refinement bits must be enclosed. The $B$ bits header includes the initial threshold value, i.e., $p$, and a word indicating the corresponding base. The integer $p$ is encoded with a fixed number of bits equal to the resolution of the samples of the database. Relating to the wavelet base, by maintaining a table with the decomposition bases, the length of the word will depend on the amount of possible bases $A_{n}$ given by Eq. (4). Thus, the number of bits $H$ to represent the word that indicates the WP filter bank can be calculated as

$H=\left\lceil\log _{2}\left(A_{n}\right)\right\rceil$,

where $\lceil\cdot\rceil$ denotes rounding to the next larger integer.

\subsection{Embedded filter bank ( $E F B$ ) algorithm}

The basic idea of the above algorithm is to encode a significant map without considering any zerotree, i.e., without taking into account the hierarchies in the WP transform. Thus, the resulting alphabet that encodes the significance map consists of three symbols (Fig. 4). This simplification allows us to use the quantization algorithm with N-PR CMFB, thereby giving another compression scheme. The N-PR CMFB-based quantization algorithm is exactly the same as that above for the WP decomposition case. Only two things must be taken into account so as to properly reinterpret the complete algorithm:

- The $\left\{c_{i}\right\}, \forall i=1, \ldots, N$ are now the set of the $N$ samples of the subband signals $x_{k}[n]$ given by the N-PR CMFB.

- The header of the bit stream in Fig. 6 only includes the initial threshold.

\section{Experimental studies}

In this section, simulations are provided to show the performance of the proposed algorithm. First, we compare it against the conventional EZW compression algorithm [7,13]. Subsequently, we carry out new experiments to show the performance

$S$ bits

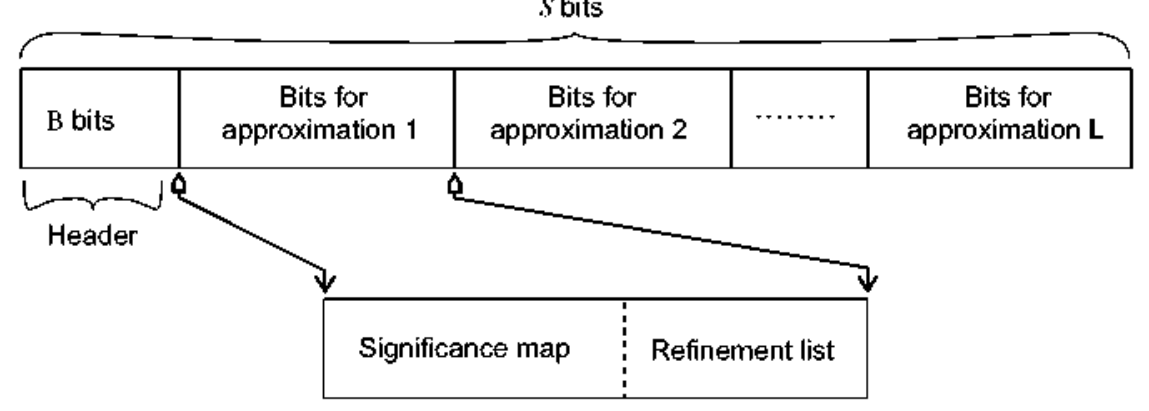

Fig. 6. Coding of a block. 
of the proposed methods compared to approaches reported in other works.

All the signals used in the examples are from the MIT-BIH Arrhythmia Database . Every file in the database consists of two lead recordings sampled at $360 \mathrm{~Hz}$ with 11 bits per sample of resolution. A baseline of 1024 has been added to each ECG for storage purposes that is removed before processing.

\subsection{Specifications of decomposition methods}

WP and N-PR CMFB were studied for the design of a thresholding-based ECG compressor

Both decomposition structures, which are implemented as filter banks, were designed to be equivalent. Therefore, to accomplish the evaluation of the method proposed in this work, the design of the decomposition methods is based on that reported in [11], which is as follows:

- The number of layers for DWT and WP are up to 4.

- The N-PR CMFB chosen is a 191-order 16-channel filter bank, using the Blackman window for designing the prototype filter.

- Based on the good results reported by other authors $[7,8]$, we utilize the Cohen-DaubechiesFeauveau 9/7 (bior9.7).

- The number of samples $N$ per block of incoming signal is 1024 which is the size most commonly used by other authors $[7,8,10]$.

\subsection{Performance measturement}

Physicians analyze the ECG by visual inspection looking for specific patterns that can be related to pathologies. Therefore, the quality of the reconstructed ECG after compressing relies on visual features. When dealing with the reconstructed ECG, signal morphology and waveform have to be as close as possible to the original so that all the pathological information remains and no additional distortion that could misunderstand a correct diagnosis is included. Therefore, the quality in ECG compression is assessed as the degree of similarity between the original signal and the retrieved signal. This is carried out using an objective parameter. The percentage root-meansquare difference (PRD) is an accepted criterion for the performance measurement of an ECG compressor . Accordingly, let $\mathbf{x}$ and $\hat{\mathbf{x}}$ be the
$N$-dimensional vectors representing the original and reconstructed signals, respectively. The PRD is defined as

$\operatorname{PRD}=(\|\mathbf{x}-\hat{\mathbf{x}}\| /\|\mathbf{x}\|) \cdot 100$,

where $\|\cdot\|$ denotes the Euclidean or $l^{2}$-norm. This parameter depends on the mean value of the original signal. Even if the 1024-baseline is removed from ECGs of the MIT-BIH Arrhythmia Database, a dc level remains. It is thus strongly recommended that the following criteria be used

PRD1 $=(\|\mathbf{x}-\hat{\mathbf{x}}\| /\|\mathbf{x}-\bar{x}\|) \cdot 100$,

where $\bar{x}$ is the mean value of the signal. It was established in [21] for the MIT database a classification of 'very good and 'good for reconstructed ECGs when PRD1 is within the ranges $0-2$ and 2-9, respectively. As a result, PRD1 values under 9 correspond to good, or very good, results.

As the incoming signal is split into segments of 1024 samples ( $N=1024$ ), the $C R$ can be calculated as

$\mathrm{CR}=\frac{N \times 11}{S}$,

where $S$ is the bit stream length for every input block (Fig. 6).

\subsection{Comparison against the conventional $E Z W$ algorithm}

\subsubsection{Entropy coding}

Here, a run-length code is designed to entropy encode the significance map (Fig. 6). For both methods proposed in this work, a bit ' 1 ' marks a significant coefficient so that the following indicates the sign: ' 11 ' is used for $\{P O S\}$ and '10' for $\{\mathrm{NEG}$. Non-significant coefficients corresponding to the symbol $\{I Z\}$ are marked by ' 0 ' and are run-length encoded. Every time $\{I Z\}$ appears, the next $B_{1}$ bits are used to encode the number of consecutive $\{\mathrm{IZ}\}$ symbols. In case of overflow, $B_{2}$ more bits are used. Therefore, a run-length of $2^{B_{1}}+2^{B_{2}}-1$ consecutive symbols can be encoded. In this work, $B_{1}=5$ and $B_{2}=\log _{2} N$, where $N$ is the total number of samples in the block.

To encode the significance map of the conventional EZW algorithm, note that the alphabet of the significance map has four symbols, and 2 bits are used for each symbol. Both $\{Z T R\}$ and $\{I Z\}$ symbols are run-length encoded as explained above, where $B_{1}$ and $B_{2}$ are 2 and 8 , respectively. The bit stream 
for every incoming block is as in Fig. 6, but as EZW algorithm utilizes the DWT, no word indicating the base has to be included in the header.

\subsubsection{Simulations}

The experiment is carried out over both 10 -min long leads extracted from records $100,101,102$, $103,107,109,111,115,117,118$ and 119 from the MIT-BIH Arrhythmia Database. This data set was proposed and it consists of a variety of signals with different rhythms, QRS complex morphologies and ectopic beats. Fig. 7 shows the performance of the proposed compressors compared with the conventional EZW. The CR is in the horizontal

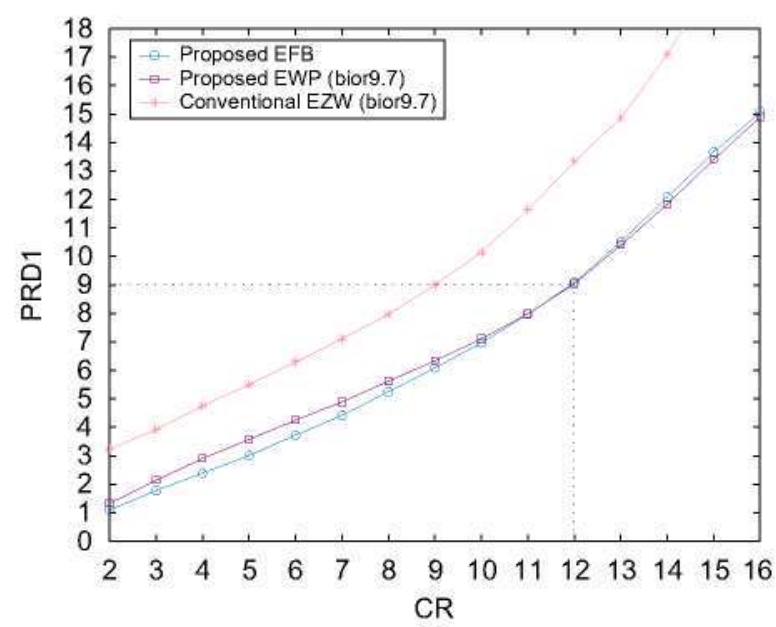

Fig. 7. Results from the first experiment showing PRD1 against CR.

a

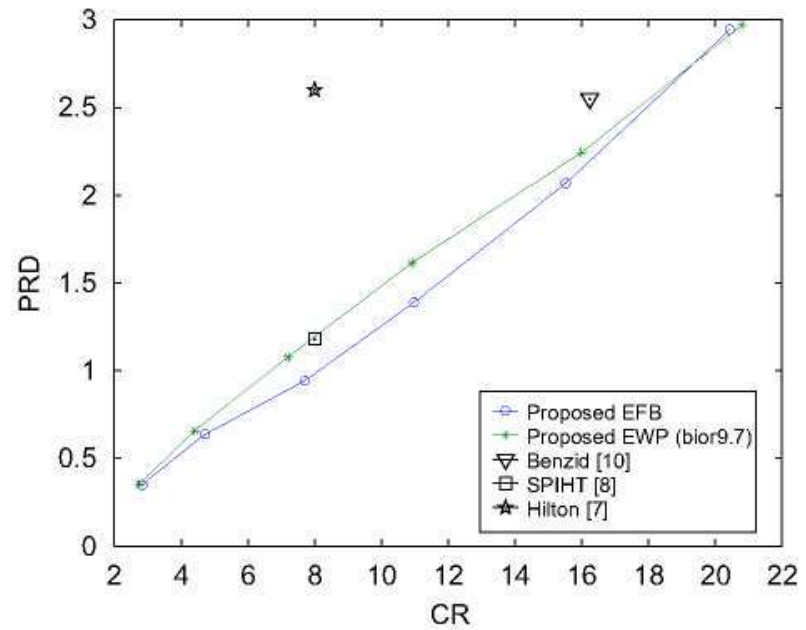

axis because it is the target parameter and can be considered as the independent variable. Both the WP and N-PR CMFB-based compressors (referred to as EWP and EFB, respectively, in Fig. 7) yields improved performance over the conventional EZW technique in the full range of CR values. On the other hand, both proposed compression methods perform similar, though for PRD1 values under $9 \%$, the method with N-PR CMFB yields better results. It is important to emphasize that although N-PR CMFB does not fulfill the PR constraint, it performs better in the range of the high quality values. The main reason for this performance is that the filters of the N-PR CMFB have higher discrimination and selectivity to keep every subband signal more independent of each other. Thus any processing accomplished at any subband signal has lower influence to the others.

\subsection{Comparison with other works}

For the purpose of comparison against other approaches, Huffman coding is used as entropy coder instead of the run-length coding since the former gives higher CR. Both the significance map and the RL are Huffman encoded [22]. The comparison is depicted in Fig. 8 over the first 1-min long lead of the records 117 and 232. The results reported from other representative works for those signals are included in the graphics. In this case, we do not consider any target parameter such as the CR or the PRD, so the compression finishes

b

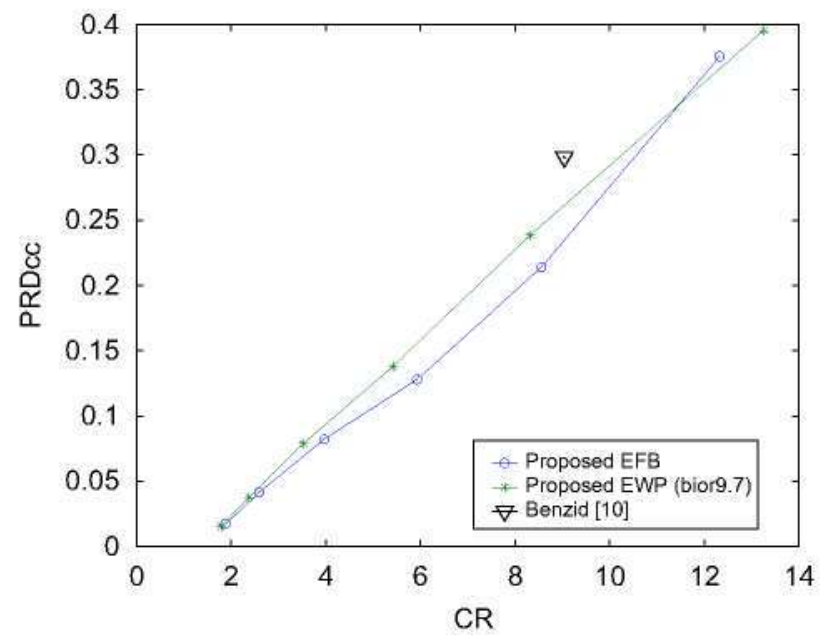

Fig. 8. Comparison against results reported by other representative works. (a) Record 117. (b) Record 232. 
after a specific number of iterations. Accordingly, our algorithm has been successively run to provide the results achieved after iterations ranging from 5 to 10. The evaluation of PRD against CR is shown.
In Fig. 8(a), the vertical axis gives the PRD obtained with Eq. (9) after removing the 1024baseline while in Fig. 8(b), the PRD (called here PRDcc) obtained with Eq. (9) but with the
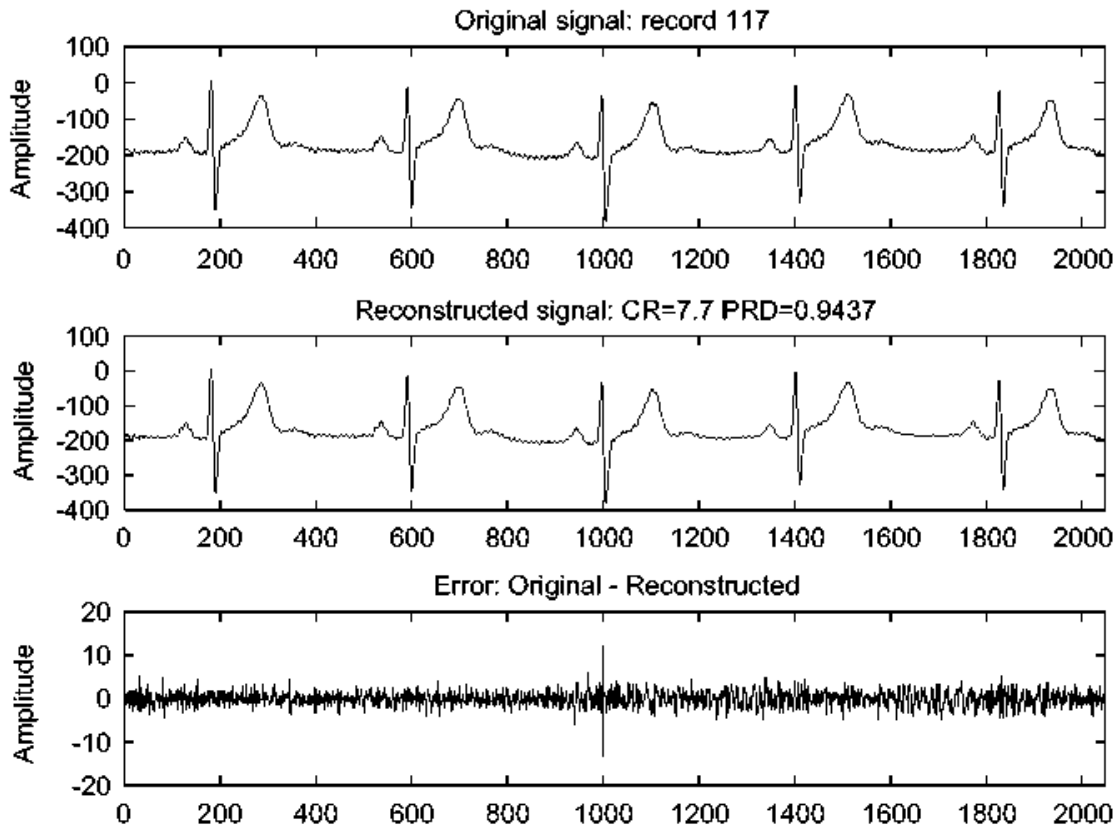

Fig. 9. Compression waveform of record 117 for $C R=7.7$ and $P R D=0.9437$.
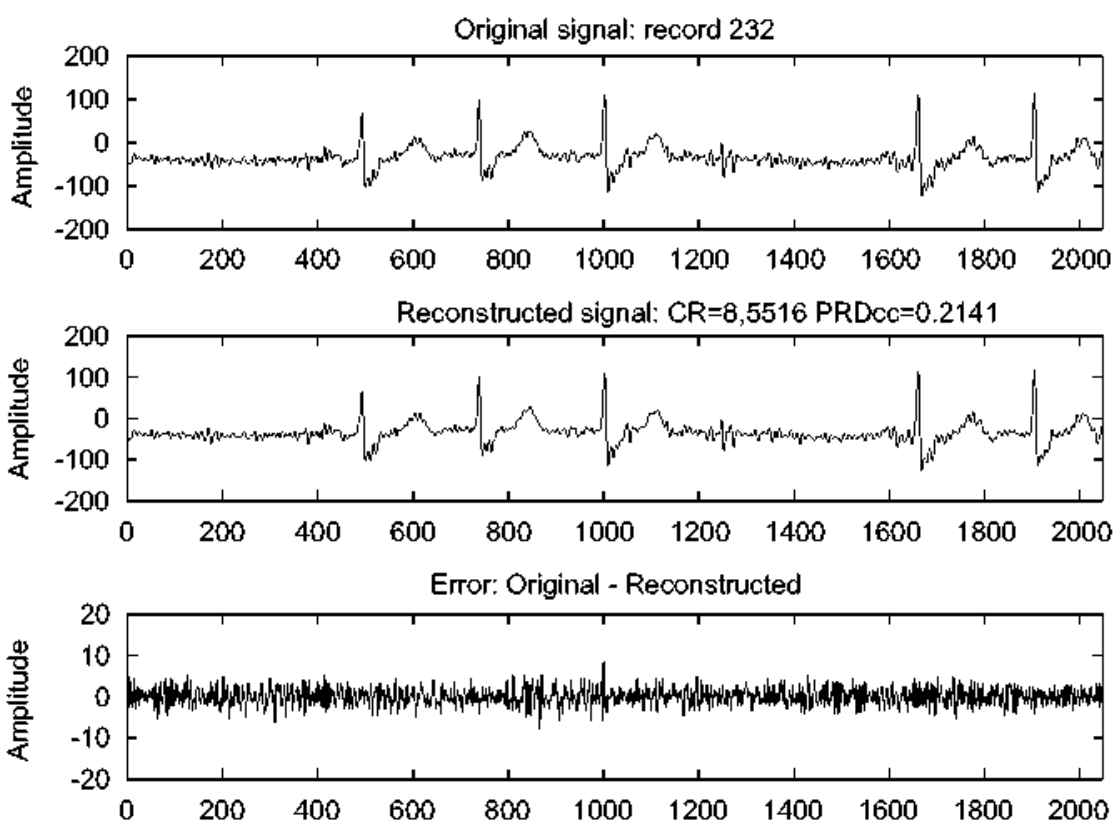

Fig. 10. Compression waveform of record 232 for $\mathrm{CR}=8.55$ and $\mathrm{PRD} \propto \mathrm{C}=0.2141$. 


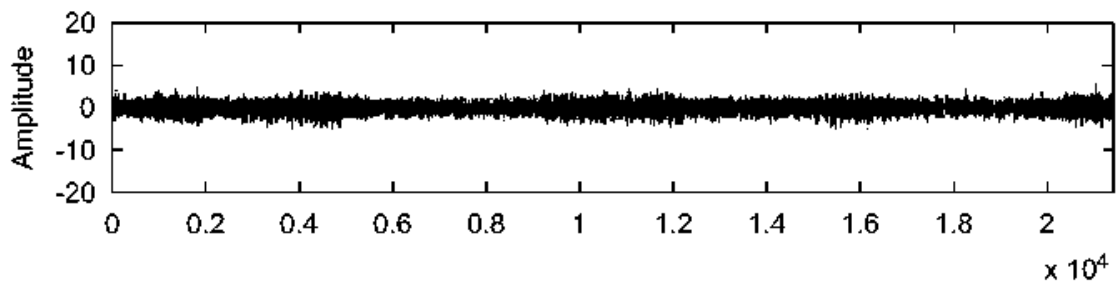

Fig. 11. Error signal for 1-min long of record $117(C R=7.7)$.

corresponding baseline included is shown. As can be seen, the proposed method with N-PR CMFB yields comparable CRs.

\subsection{Vistal study}

The waveforms given by the proposed compression scheme visually evaluated in Figs. 9 and 10 where depicted are the first 2048 samples of the original signal, the reconstructed signal and the error signal (from top to bottom) of the records 117 and 232 whose compression values are those of Fig. 8. The waveforms are obtained with the N-PR CMFB-based compressor. In both cases, the reconstructed signals remain close to the original signals and the error signals are equally distributed along the horizontal axis.

The isolated high local error value around $n=$ 1000 in Fig. 9 is considered as the distortion between consecutive blocks of incoming signals. However, Fig. 11 depicts the error over the full range of samples and, as remarked above, the error is equally distributed along the axis with only isolated large samples.

\section{Conclusions}

In this paper, a new approach for the design of embedded ECG compressors based on filter banks is given. In the quantization stage, zerotrees are not encoded reducing thus the alphabet of the significance map to three symbols. The new approach has two main advantages over the traditional EZW algorithm: (1) it is a simpler algorithm and (2) it can be utilized with many other kind of filter banks than the corresponding to DWT.

Two compressors are proposed with this quantization algorithm:

(1) The embedded wavelet packets (EWP) algorithm that makes use of WP to decompose the incoming ECG.
(2) The embedded filter bank (EFB) algorithm that utilizes N-PR CMFB to represent the original ECG.

Simulations show that: (a) both compressors perform better than the traditional EZW applied in the DWT domain and (b) the EFB algorithm yields better performance than the EWP within the high quality range for the retrieved ECG, i.e., the range of PRD1 below 9\%. When compared to other authors, both schemes provide compression results comparable to those of the state of the art. Again, the EFB method outperforms the EWP method.

The proposed methods show an efficient performance and are computationally simple

. The N-PR

CMFB-based scheme demonstrates better performance than the WP-based scheme. Other compression schemes with N-PR CMFB have previously reported good performance . Therefore, given that N-PR CMFB admits efficient real-time implementation [11] and that the N-PR CMFB-based scheme outperforms the WP-based scheme, it can be considered as an appropriate tool for ECG compression.

\section{Acknowledgments}

This work has been supported in part by Grant PR-2007-0216 from the Ministerio de Educación y Ciencia, in part by the Fondo de Investigación Sanitaria under Project PI052277, and in part by Comunidad Autónoma de Madrid and Universidad de Alcalá through project CCG06-UAH/TIC-0417.

\section{References}

N.J. Holter, New methods for heart studies. Science 134 (1961) 1214-1220.

K. Hung, Y,-T. Zhang, Implementation of a WAP-based telemedicine system for patient monitoring, IEEE Trans. Inform. Technol. Biomed. 7 (2) (June 2003) 101-107. 
C.H. Salvador, M.P. Carrasco. M.A.G. de Mingo, A.M. Carrero, J.M. Montes, L.S. Martín. M.A. Cavero, I.F. Lozano, J.L. Monteagudo. Airmed-cardio: a GSM and internet services-based system for ont-of-hospital follow-up of cardiac patients. IEEE Trans. Inform. Technol. Biomed. 9 (1) (March 2005) 73-84.

J. Rodríguez A. Goñi, A. Illarramendi, Real-time classification of ECGs on a PDA. IEEE Trans. Inform. Technol. Biomed. 9 (1) (March 2005) 23-34.

L. Senhadji, J.J. Bellanger, G. Garrault, J.L. Coatrieux. Wavelet analysis of ECG signals, in: Proceedings of the 12th Annual International Conference on the IEEE Engineering in Medicine and Biology, vol. 12. 1990, pp. 811-812.

A. Djohan, T.Q. Nguyen. W.J. Tompkins. ECG compression using discrete symmetric wavelet transform, in: Proceedings of the 17th Annual International Conference on the IEEE Engineering in Medicine and Biology. vol. 1. 1995, pp, 167-168,

M.L. Hilton. Wavelet and wavelet packets compression of electrocardiogram, IEEE Trans. Biomed. Eng. 44 (5) (May 1997) $394-402$.

Z. Lu. D.Y. Kim, W.A. Pearlman. Wavelet compression of ECG signals by the set partitioning in hierarchical trees algorithm, IEEE Trans. Biomed. Eng. 47 (7) (July 2000) 849-856.

Y. Zigel. A. Cohen, A. Katz. ECG signal compression using analysis by synthesis coding. IEEE Trans. Bioned. Eng. 47 (10) (October 2000) 1308-1313.

R. Benzid, F. Marir. A. Boussaad, M. Benyoucef, D. Arar, Fixed percentage of wavelet coefficients to be zeroed for ECG compression, Electron. Lett. 39 (11) (May 2003) $830-831$.

M. Blanco-Velasco, F. Cruz-Roldán. F. López, Á.M. Bravo, D. Martinez, A low computational complexity algorithn for ECG signal compression. Medical Eng. Phys. 26 (7) (September 2004) 553-568.
M. Blanco-Velasco, F. Cruz-Roldán, J.I. Godino-Llorente, K.E. Baner. ECG compression with retieved quality guaranteed. Electron. Lett. 40 (23) (November 2004) 1466-1467.

J.M. Shapiro, Embedded image coding using zerotrees of wavelet coefficients. IEEE Trans. Signal Process. 41 (12) (December 1993) 3445-3462.

J.A. Norris, K.B. Englehart, D.F. Lovely, Myoelectric signal compression using zerotrees of wavelets coefficients. Medical Eng. Phys. 25 (9) (November 2003) 739-746.

G. Strang, T.Q. Nguyen. Wavelets and Filter Banks, Wellesley-Cambridge. Wellesley. MA, 1996.

R.R. Coifman, M.V. Winckerhauser, Entropy-based algorithns for best basis selection. IEEE Trans. Inform. Theory 38 (2) (March 1992) 713-718.

F. Cruz-Roldán, P. Amo-López, S. Maldonado, S.S. Lawson, An efficient and simple method for designing prototype filters for cosine-modulated psendo-QMF banks, IEEE Signal Process. Lett. 9 (1) (January 2002) 29-31.

P.P. Vaidyanathan. Multirate Systems and Filter Banks, Prentice-Hall, Englewood Cliffs, NJ, 1993.

F. Cruz-Roldán, M. Monteagudo-Prim, Efficient implementation of nearly-perfect FIR reconstruction cosine-modulated filter banks, IEEE Trans. Signal Process. 52 (9) (September 2004) 2661-2664.

A.L. Goldberger. L.A.N. Amaral. L. Glass. J.M. Hausdorff, P.C. Ivanov. R.G. Mark, J.E. Mietus. G.B. Moody. C.-K. Peng. H.E. Stanley. PhysioBank, PhysioToolkit. and PhysioNet: components of a new research resource for complex physiologic signals, Circulation 101 (23) (June 2000) 215-220. Y. Zigel. A. Cohen, A. Katz, The weighted diagnostic distortion (WDD) measure for ECG signal compression, IEEE Trans. Biomed. Eng. 47 (11) (November 2000) 1422-1430.

K. Skretting. J. Hakon, S.O. Aase, Inproved Huffman coding using recursive splitting. in: Proceedings of NORSIG 99. September 1999. 